Pacific

Journal of

Mathematics

A BIRATIONAL INVARIANT FOR ALGEBRAIC GROUP ACTIONS

Z. ReichSTEIN AND B. Youssin

Volume $204 \quad$ No. 1

May 2002 


\title{
A BIRATIONAL INVARIANT FOR ALGEBRAIC GROUP ACTIONS
}

\author{
Z. Reichstein And B. YOUSSIN
}

\begin{abstract}
We construct a birational invariant for certain algebraic group actions. We use this invariant to classify linear representations of finite abelian groups up to birational equivalence, thus answering, in a special case, a question of E.B. Vinberg and giving a family of counterexamples to a related conjecture of P.I. Katsylo. We also give a new proof of a theorem of $M$. Lorenz on birational equivalence of quantum tori (in a slightly expanded form) by applying our invariant in the setting of $\mathrm{PGL}_{n}$-varieties.
\end{abstract}

\section{Introduction.}

Let $G$ be an algebraic group and let $X$ be a smooth projective $G$-variety (i.e., an algebraic variety with a $G$-action) defined over an algebraically closed base field of characteristic zero. It is shown in $[\mathbf{R Y} \mathbf{1}]$ that for each finite abelian subgroup $H$, the presence of an $H$-fixed points is a birational invariant of $X$ as a $G$-variety. In other words, if $X$ and $Y$ are birationally isomorphic smooth projective $G$-varieties and $H$ is a finite abelian subgroup of $G$ then $X^{H} \neq \emptyset$ iff $Y^{H} \neq \emptyset$. (Here as usual, $X^{H}$ denotes the subvariety of $H$-fixed points of $X$.) Note that only nontoral finite abelian subgroups $H$ are of interest in this setting; if $H$ lies in a torus $T$ of $G$ then $X^{T}$ (and thus $X^{H}$ ) can never be empty by the Borel Fixed Point theorem. In [RY1], [RY2] and [RY3] we used $H$-fixed points for nontoral finite abelian subgroup $H$ of $G$ to study the geometry of $G$-varieties (their essential dimensions, splitting degrees, etc.) and properties of related algebraic objects (field extensions, division algebras, octonion algebras, etc.).

In this paper we will associate (under additional assumptions on $X, G$ and $H)$ a more subtle invariant $i(X, x, H)$ to a point $x \in X^{H}$; the precise definition is given in Section 4. Our main result about $i(X, x, H)$ is stated below.

Recall that the rank of a finite abelian group $H$ is the minimal number of generators of $H$ (see Section 2) and that a $G$-variety $X$ is called generically free if $\operatorname{Stab}(x)=\{1\}$ for $x$ in general position in $X$ (see Section 3 ).

Theorem 1.1. Let $G$ be an algebraic group of dimension $d, H$ be a finite abelian subgroup of $G$ of rank $r$, and $X, Y$ be birationally isomorphic smooth 
projective irreducible generically free $G$-varieties of dimension $d+r$. Assume that $\operatorname{Stab}(x)$ is finite for every $x \in X^{H}$ and $\operatorname{Stab}(y)$ is finite for every $y \in Y^{H}$. Then for every $x \in X^{H}$ there exists a $y \in Y^{H}$ such that $i(Y, y, H)=$ $i(X, x, H)$.

Informally speaking, the presence of $H$-fixed points $x$ with a prescribed value $i(X, x, H)$ (on a suitable model) is a birational invariant of $X$ as a $G$ variety. Our proof of Theorem 1.1, presented in Section 6, relies on canonical resolution of singularities. Note that Theorem 1.1 remains valid even if $X$ and $Y$ are not assumed to be irreducible; see Remark 6.4.

We give two applications of Theorem 1.1.

A birational classification of linear representations. For our first application recall that by the no-name lemma any two generically free linear representations of a given algebraic group $G$ are stably isomorphic as $G$ varieties; see, e.g., $[\mathbf{P}, 1.5 .3]$. Thus it is natural to try to classify such representations up to birational isomorphism. This problem was proposed by E. B. Vinberg [PV2, pp. 494-496]; see also [P, 1.5.1]. P. I. Katsylo has subsequently stated the following conjecture:

Conjecture $1.2([\mathbf{K}]$; see also $[\mathbf{P}, 1.5 .10])$. Let $V$ and $W$ be generically free linear representations of an algebraic group $G$. If $\operatorname{dim}(V)=\operatorname{dim}(W)$ then $V$ and $W$ are birationally isomorphic as $G$-varieties.

In this paper we will establish the following birational classification of faithful linear representations of a diagonalizable group. Recall that every diagonalizable group $G$ can be uniquely written in the form

$$
\begin{aligned}
G= & \mathbb{G}_{m}\left(n_{1}\right) \times \cdots \times \mathbb{G}_{m}\left(n_{r}\right) \quad \text { such that } \\
& \mathbb{G}_{m}\left(n_{1}\right) \subset \cdots \subset \mathbb{G}_{m}\left(n_{r}\right) \quad \text { and each } n_{i}=0 \text { or } \geq 2,
\end{aligned}
$$

where $\mathbb{G}_{m}=k^{*}=\mathbb{G}_{m}(0)$ denotes the 1-dimensional torus, $\mathbb{G}_{m}(n) \simeq \mathbb{Z} / n \mathbb{Z}$ is the $n$-torsion subgroup of $\mathbb{G}_{m}$, and $\mathbb{G}_{m}(a) \subset \mathbb{G}_{m}(b)$ iff either $a$ divides $b$ or $a=b=0$; see, e.g., [Bo, Proposition III.8.7] and [DF, Theorem 5.2.3]. Recall also that a representation of a diagonalizable group is faithful iff it is generically free ([PV1, Proposition 7.2]) and that any such representation has dimension $\geq r$ ([Bo, Proposition III.8.2(d)]).

Theorem 1.3. Let $G$ be a diagonalizable group, as in (1.1).

(a) If $d \geq r+1$ then any two faithful d-dimensional linear representations of $H$ are birationally equivalent.

(b) If $n_{1}=0$ or 2 then any two faithful $r$-dimensional linear representations of $H$ are birationally equivalent.

(c) If $n_{1} \geq 3$ then $H$ has exactly $\phi\left(n_{1}\right) / 2$ birational equivalence classes of faithful r-dimensional representations. Here $\phi$ denotes the Euler $\phi$-function. 
In particular, Conjecture 1.2 fails for $G$ if and only if $n_{1}=5$ or $\geq 7$.

The birational equivalence classes of faithful linear representations of $G$ are explicitly described in Theorem 7.1. Later in Section 7 we will show that Conjecture 1.2 also fails for some nonabelian finite groups $G$. On the other hand, we remark that P. I. Katsylo $[\mathbf{K}]$ proved Conjecture 1.2 for $G=\mathrm{SL}_{2}$, $G=\mathrm{PGL}_{2}$ and $G=S_{n}(n \leq 4)$, and that many interesting cases remain open, including $G=\mathrm{S}_{n}(n \geq 5)$ and $G=$ arbitrary connected semisimple group.

Birational equivalence of quantum tori. Our second application is based on the fact that birational isomorphism classes of generically free $\mathrm{PGL}_{n}$-varieties are in natural correspondence with central simple algebras of degree $n$; see, e.g., [Se1, X.5] or [RY2, Section 3]. Thus Theorem 1.1 (with $G=\mathrm{PGL}_{n}$ ) will sometimes allow us to prove that certain division algebras are not isomorphic over $k$.

Let $\omega_{1}, \ldots, \omega_{r}$ be roots of unity and let $R\left(\omega_{1}, \ldots, \omega_{r}\right)$ be the associative $k$-algebra $k\left\{x_{1}^{ \pm 1}, \ldots, x_{2 r}^{ \pm 1}\right\}$, where the variables $x_{1}, \ldots, x_{2 r}$ are subject to relations $x_{2 i-1} x_{2 i}=\omega_{i} x_{2 i} x_{2 i-1}$ for $i=1, \ldots, r$ and $x_{a} x_{b}=x_{b} x_{a}$ for all other pairs $x_{a}, x_{b}$. Denote the algebra of quotients of $R\left(\omega_{1}, \ldots, \omega_{r}\right)$ by $Q\left(\omega_{1}, \ldots, \omega_{r}\right)$. Note that $Q\left(\omega_{1}, \ldots, \omega_{r}\right)$ is obtained from $R\left(\omega_{1}, \ldots, \omega_{r}\right)$ by adjoining the inverses of all central elements and that $Q\left(\omega_{1}, \ldots, \omega_{r}\right)$ is a finite-dimensional division algebra (in fact, it is a tensor product of symbol algebras).

M. Lorenz [Lo1, Proposition 1.3] showed that $Q(\omega)$ and $Q\left(\omega^{\prime}\right)$ are isomorphic as $k$-algebras if and only if $\omega^{\prime}=\omega^{ \pm 1}$. In Section 8 we will give a geometric proof of the following variant of this result.

Theorem 1.4. Suppose $\omega_{i}$ is a primitive $n_{i}$ th root of unity, $n_{i}$ divides $n_{i+1}$ for $i=1, \ldots, r-1, n_{1} \geq 2$, and $\left(m_{i}, n_{i}\right)=1$. Then $Q\left(\omega_{1}, \ldots, \omega_{r}\right)$ and $Q\left(\omega_{1}^{m_{1}}, \ldots, \omega_{r}^{m_{r}}\right)$ are isomorphic as $k$-algebras if and only if $m_{1} \cdots m_{r} \equiv \pm 1$ $\left(\bmod n_{1}\right)$.

The centers of the algebras $Q\left(\omega_{1}, \ldots, \omega_{r}\right)$ and $Q\left(\omega_{1}^{m_{1}}, \ldots, \omega_{r}^{m_{r}}\right)$ can be naturally identified with the field $K=k\left(x_{1}^{n_{1}}, \ldots, x_{2 r}^{n_{2} r}\right)$. Note that these algebras may be $k$-isomorphic but not $K$-isomorphic, i.e., not Brauer equivalent. More precisely, $Q\left(\omega_{1}, \ldots, \omega_{r}\right)$ and $Q\left(\omega_{1}^{m_{1}}, \ldots, \omega_{r}^{m_{r}}\right)$ are Brauer equivalent iff $m_{i} \equiv 1\left(\bmod n_{i}\right)$ for every $i=1, \ldots, r$.

It is natural to think of $R\left(\omega_{1}, \ldots, \omega_{r}\right)$ and $Q\left(\omega_{1}, \ldots, \omega_{r}\right)$ as, respectively, the "coordinate ring" and the "function field" of a quantum torus. Using this terminology, one may view Theorem 1.4 as a result about birational isomorphism classes of quantum tori.

Finally we remark that M. Lorenz has communicated to us a proof of Theorem 1.4 based on the techniques of [Lo1] and [Lo2]. His argument works in arbitrary characteristic. 
Acknowledgements. We are grateful to P.I. Katsylo, M. Lorenz and V.L. Popov for helpful communications related to the subject matter of this paper.

The second author warmly thanks Institute of Mathematics of Hebrew University for its hospitality during 1999/2000.

\section{Linear algebra in abelian groups.}

Recall that any finitely generated abelian group $(A,+)$ can be written in the form

$$
\begin{gathered}
A \simeq \mathbb{Z} / n_{1} \mathbb{Z} \times \cdots \times \mathbb{Z} / n_{r} \mathbb{Z}, \text { where each } n_{i}=0 \text { or } \geq 2 \\
\text { and } n_{i+1} \in n_{i} \mathbb{Z} \text { for every } i=1, \ldots, r ;
\end{gathered}
$$

see, e.g., [DF, Theorem 5.2.3]. Here $r$ and $n_{1}, \ldots, n_{r}$ are uniquely determined by the isomorphism type of $A$. We shall refer to the integer $r$ as the the rank of $A$; equivalently, the rank of $A$ equals the minimal possible number of generators of $A$.

Recall that if $B$ is an abelian group then the dual group $B^{*}$ is defined as $\operatorname{Hom}(B, \mathbb{Q} / \mathbb{Z})$; we will often identify $\mathbb{Q} / \mathbb{Z}$ with the multiplicative group of roots of unity in $k$. The finitely generated group $A$ of (2.1) and the diagonalizable group $G$ of (1.1) are dual to each other. The rank of a diagonalizable group $G$ is defined as the rank of the finitely generated group $G^{*}$ (in particular, the group $G$ of (1.1) has rank $r$ ). Note that this is consistent with the way we defined rank for a finitely generated group: Indeed, if $A$ is both diagonalizable and finitely generated, i.e., is finite abelian, then $A$ and $A^{*}$ are isomorphic, so that their ranks coincide.

Skew-symmetric powers. We will write $\bigwedge^{d}(A)$ for the $d$-th skew-symmetric power of $A$, viewed as a $\mathbb{Z}$-module.

The proof of the following lemma is elementary; we leave it as an exercise for the reader.

Lemma 2.1. Let $A$ be a finitely generated abelian group as in (2.1). Then

(a) $\bigwedge^{r}(A) \simeq \mathbb{Z} / n_{1} \mathbb{Z}$.

(b) $\bigwedge^{d}(A)=(0)$, if $d \geq r+1$.

Definition 2.2. Let $A$ be a finite abelian group. Let $\omega: A \times A \longrightarrow \mathbb{Q} / \mathbb{Z}$ be a $\mathbb{Z}$-bilinear form. As usual, we shall say that

(a) $\omega$ is alternating if $\omega(a, a)=0$ for every $a \in A$,

(b) $\omega$ is nondegenerate if $\omega(a, \cdot)$ is not identically zero for any $a \in A-\{0\}$,

(c) $\omega$ is symplectic if it is both alternating and nondegenerate.

Lemma 2.3. Let $A$ be a finite abelian group of rank $2 r, \omega$ be a symplectic form of $A$, and $\psi$ be an $\omega$-preserving automorphism $A \longrightarrow A$. Then

(a) $\wedge^{2 r} \psi$ is the trivial automorphism of $\bigwedge^{2 r}(A)$ and 
(b) $\wedge^{2 r} \psi^{*}$ is the trivial automorphism of $\bigwedge^{2 r}\left(A^{*}\right)$.

Proof. (a) It is well-known that $A$ can be written in the form $A=A_{0} \oplus A_{0}^{*}$ such that

$$
\omega\left(\left(a, a^{*}\right),\left(b, b^{*}\right)\right)=a^{*}(b)-b^{*}(a)
$$

for any $a, b \in A_{0}$ and $a^{*}, b^{*} \in A_{0}^{*}$; see, e.g., [TA, Theorem 4.1]. Write $A_{0}$ as $\mathbb{Z} / n_{1} \mathbb{Z} \times \cdots \times \mathbb{Z} / n_{r} \mathbb{Z}$, where $n_{i}$ divides $n_{i+1}$ for $i=1, \ldots, r-1$ and $n_{1} \geq 2$. Let $e_{i} \in A_{0}$ be a generator of the of the $i$ th factor, and let $f_{i} \in A_{0}^{*}$ be given by $f_{i}: A_{0} \longrightarrow \mathbb{Z} / n_{i} \mathbb{Z} \longrightarrow \mathbb{Q} / \mathbb{Z}$, where the first map the projection to the $i$ th factor, and the second map takes $e_{i}$ to $1 / n_{i}$. Then every $a \in A$ can be written in the form $a=\sum_{i=1}^{n}\left(\alpha_{2 i-1} e_{i}+\alpha_{2 i} f_{i}\right)$, where $\alpha_{2 i-1}, \alpha_{2 i} \in \mathbb{Z}$, and (2.2) translates into

$$
\begin{aligned}
& \omega\left[\sum_{i=1}^{n}\left(\alpha_{2 i-1} e_{i}+\alpha_{2 i} f_{i}\right), \sum_{i=1}^{n}\left(\beta_{2 i-1} e_{i}+\beta_{2 i} f_{i}\right)\right] \\
& =\sum_{i=1}^{n} \frac{1}{n_{i}}\left(\alpha_{2 i} \beta_{2 i-1}-\alpha_{2 i-1} \beta_{2 i}\right) .
\end{aligned}
$$

Suppose

$$
\begin{aligned}
& \psi\left(e_{1}\right)=c_{11} e_{1}+c_{12} f_{1}+\cdots+c_{1,2 r-1} e_{r}+c_{1,2 r-1} f_{r}, \\
& \psi\left(f_{1}\right)=c_{21} e_{1}+c_{22} f_{1}+\cdots+c_{2,2 r-1} e_{r}+c_{2,2 r} f_{r}, \\
& \vdots \\
& \psi\left(e_{r}\right)=c_{2 r-1,1} e_{1}+c_{2 r-1,2} f_{1}+\cdots+c_{2 r-1,2 r-1} e_{r}+c_{2 r-1,2 r} f_{r}, \\
& \psi\left(f_{r}\right)=c_{2 r, 1} e_{1}+c_{2 r, 2} f_{1}+\cdots+c_{2 r, 2 r-1} e_{r}+c_{2 r, 2 r} f_{r},
\end{aligned}
$$

where $C=\left(c_{i j}\right)_{i, j=1, \ldots, 2 r} \in \mathrm{M}_{n}(\mathbb{Z})$. Since $\lambda=e_{1} \wedge f_{1} \wedge \cdots \wedge e_{r} \wedge f_{r}$ generates $\bigwedge^{2 r}\left(A^{*}\right) \simeq \mathbb{Z} / n_{1} \mathbb{Z}$ and $\left[\wedge^{2 r} \psi\right](\lambda)=\operatorname{det}(C) \lambda$, it is sufficient to show that $\operatorname{det}(C)=1\left(\bmod n_{1}\right)$.

The condition that $\psi$ preserves $\omega$ translates into $C J C^{t}=J(\bmod 1)$, where $C^{t}$ is the transpose of $C$ and

$$
J=\left(\begin{array}{ccccc}
0 & 1 / n_{1} & \cdots & 0 & 0 \\
-1 / n_{1} & 0 & \cdots & 0 & 0 \\
\vdots & & \vdots & & \vdots \\
0 & 0 & & 0 & 1 / n_{r} \\
0 & 0 & & -1 / n_{r} & 0
\end{array}\right)
$$

In other words,

$$
C J C^{t}=J+N,
$$

where $N$ is a skew-symmetric integral matrix. We shall deduce the desired equality, $\operatorname{det}(C)=1\left(\bmod n_{1}\right)$, by computing the Pfaffian on both sides 
of (2.3). On the one hand

$$
\operatorname{Pf}\left(C J C^{t}\right)=\operatorname{det}(C) \operatorname{Pf}(J)=(-1)^{r} \operatorname{det}(C) \frac{1}{n_{1} \ldots n_{r}} ;
$$

see, e.g., [Lang, XIV, 10, Theorem 7]. On the other hand, suppose $X=$ $\left(x_{i j}\right)$, where $x_{j i}=-x_{i j}$ for $1 \leq i, j \leq 2 r$. Then $\operatorname{Pf}(X) \in \mathbb{Z}\left[x_{i j} \mid 1 \leq i<j \leq\right.$ $2 r]$ has degree 1 in every $x_{i j}$, where $i<j$. (Indeed, $\operatorname{det}(X)$ has degree 2 in every $x_{i j}$, and $\operatorname{Pf}(X)^{2}=\operatorname{det}(X)$.) Consequently,

$$
\operatorname{Pf}(J+N)=\operatorname{Pf}(J)+\frac{z}{n_{2} \ldots n_{r}}=(-1)^{r} \frac{1}{n_{1} \ldots n_{r}}+\frac{z}{n_{2} \ldots n_{r}},
$$

where $z$ is an integer. (Here we used the fact that $n_{i}$ divides $n_{i+1}$ for every $i=1, \ldots, r-1$.) Putting (2.3), (2.4) and (2.5) together, we see that $\operatorname{det}(C)=1+(-1)^{r} n_{1} z$, i.e., $\operatorname{det}(C)=1\left(\bmod n_{1}\right)$, as claimed.

(b) Let $i: A \longrightarrow A^{*}$ be the isomorphism $a \mapsto i_{a}$, where $i_{a}(b)=\omega(a, b)$. It is easy to see that the automorphism $\psi^{*}: A^{*} \longrightarrow A^{*}$ preserves the symplectic form $\omega^{*}$ on $A^{*}$ given by $\omega^{*}\left(a^{*}, b^{*}\right)=\omega\left(i^{-1} a^{*}, i^{-1} b^{*}\right)$. Applying part (a) to $\psi^{*}$, we obtain the desired result.

Elementary operations. Let $A$ be an abelian group. We will say that two $d$-tuples $\left(a_{1}, \ldots, a_{d}\right)$ and $\left(b_{1}, \ldots, b_{d}\right) \in A^{d}$ are related by an elementary operation if $\left(b_{1}, \ldots, b_{d}\right)=\left(a_{1}, \ldots, a_{i-1}, a_{i}+m a_{j}, a_{i+1}, \ldots, a_{d}\right)$ for some $i \neq j$ and $m \in \mathbb{Z}$.

Lemma 2.4. Let $a_{1}, \ldots, a_{d}$ and $b_{1}, \ldots, b_{d}$ be two sets of generators for an abelian group $A$. Then $a=\left(a_{1}, \ldots, a_{d}\right)$ and $\left(b_{1}, \ldots, b_{d}\right)$ are related by $a$ sequence of elementary operations if and only if $a_{1} \wedge \cdots \wedge a_{d}=b_{1} \wedge \cdots \wedge b_{d}$ in $\bigwedge^{d}(A)$.

Proof. It is clear that if $\left(a_{1}, \ldots, a_{d}\right)$ and $\left(b_{1}, \ldots, b_{d}\right)$ are related by a sequence of elementary operations then $a_{1} \wedge \cdots \wedge a_{d}=b_{1} \wedge \cdots \wedge b_{d}$. We will prove the converse by induction on $d$.

If $d=1$ there is nothing to prove, since $\bigwedge^{1}(A)=A$. For the induction step, assume $d \geq 2$ and $A \simeq\left(\mathbb{Z} / n_{1} \mathbb{Z}\right) \times \cdots \times\left(\mathbb{Z} / n_{r} \mathbb{Z}\right)$ is as in (2.1) Here $r=\operatorname{rank}(A) \leq d$, since we are assuming $A$ is generated by $d$ elements.

A $d$-tuple of generators $\left(a_{1}, \ldots, a_{d}\right)$ of $A$ can now be represented by a $d \times r$-matrix $a=\left(a_{i j}\right)$ whose $i$ th row is $a_{i}$. Elements of the $j$ th column of this matrix lie in $\mathbb{Z} / n_{j} \mathbb{Z}$. Elementary operation on such matrices amount to adding an integer multiple of the $j$ th row to the $i$ th row or some $i \neq j$.

Elementary operations allow us to perform the Euclidean algorithm in the last column of $\left(a_{i j}\right)$. Since $a_{1 r}, \ldots, a_{d r}$ generate $\mathbb{Z} / n_{r} \mathbb{Z}$, after a sequence 
of elementary operations, we may assume that

$$
\left(a_{i j}\right)=\left(\begin{array}{cccc} 
& & & 0 \\
& X & & \vdots \\
& & & 0 \\
* & \ldots & * & 1
\end{array}\right),
$$

where $X$ is a $(d-1) \times(r-1)$-matrix. Since the rows of $\left(a_{i j}\right)$ generate $A$, the rows of $X$ generate $\bar{A}=\left(\mathbb{Z} / n_{1} \mathbb{Z}\right) \times \cdots \times\left(\mathbb{Z} / n_{r-1} \mathbb{Z}\right)$. Thus after performing additional elementary operations, we may assume

$$
\left(a_{i j}\right)=\left(\begin{array}{cccc} 
& & & 0 \\
& X & & \vdots \\
& & & 0 \\
0 & \ldots & 0 & 1
\end{array}\right) \text {, and similarly }\left(b_{i j}\right)=\left(\begin{array}{cccc} 
& & & 0 \\
& Y & & \vdots \\
& & & 0 \\
0 & \ldots & 0 & 1
\end{array}\right) .
$$

In other words, we may assume $\left(a_{1}, \ldots, a_{d-1}\right)$ and $\left(b_{1}, \ldots, b_{d-1}\right)$ are $(d-1)$ tuples of generators in $\bar{A}$ and $a_{d}=b_{d}$ is the generator $1 \in \mathbb{Z} / n_{r} \mathbb{Z}$.

We claim that

$$
a_{1} \wedge \cdots \wedge a_{d-1}=b_{1} \wedge \cdots \wedge b_{d-1} \text { in } \wedge^{(d-1)}(\bar{A}) .
$$

Indeed, if $d \geq r+1$, this is obvious, since $\bigwedge^{d-1}(\bar{A})=(0)$; see Lemma 2.1(b). If $d=r$ then the isomorphism $\bigwedge^{d}(A) \simeq \mathbb{Z} / n_{1} \mathbb{Z}$ identifies $a_{1} \wedge \cdots \wedge a_{d}$ with $\operatorname{det}\left(a_{i j}\right)\left(\bmod n_{1}\right)$, and the isomorphism $\bigwedge^{(d-1)}(\bar{A}) \simeq \mathbb{Z} / n_{1} \mathbb{Z}$ identifies $a_{1} \wedge \cdots \wedge a_{d-1}$ with $\operatorname{det}(X)\left(\bmod n_{1}\right)$. Since $a_{1} \wedge \cdots \wedge a_{d}=b_{1} \wedge \cdots \wedge b_{d}$, we know that $\operatorname{det}\left(a_{i j}\right)=\operatorname{det}\left(b_{i j}\right)\left(\bmod n_{1}\right) ; \operatorname{hence}, \operatorname{det}(X)=\operatorname{det}(Y)\left(\bmod n_{1}\right)$, and (2.6) follows.

Now by the induction assumption $\left(a_{1}, \ldots, a_{d-1}\right)$ and $\left(b_{1}, \ldots, b_{d-1}\right)$ are related by a sequence of elementary operations. Since $a_{d}=b_{d}$, so are $\left(a_{1}, \ldots, a_{d}\right)$ and $\left(b_{1}, \ldots, b_{d}\right)$, as desired.

Corollary 2.5. Let $a_{1}, \ldots, a_{d}$ and $b_{1}, \ldots, b_{d}$ be two sets of generators for an abelian group $A$. Then the following conditions are equivalent.

(a) There exists a matrix $N=\left(n_{i j}\right) \in \mathrm{GL}_{d}(\mathbb{Z})$ such that $b_{i}=n_{i 1} a_{1}+\cdots+$ $n_{i d} a_{d}$ for $i=1, \ldots, d$.

(b) $a_{1} \wedge \cdots \wedge a_{d}= \pm b_{1} \wedge \cdots \wedge b_{d}$ in $\wedge^{d}(A)$.

Proof. The implication (a) $\Longrightarrow(\mathrm{b})$ is obvious. To prove the converse, note that we may assume without loss of generality that $a_{1} \wedge \cdots \wedge a_{d}=b_{1} \wedge$ $\cdots \wedge b_{d}$; indeed, if $a_{1} \wedge \cdots \wedge a_{d}=-b_{1} \wedge \cdots \wedge b_{d}$ then we can simply replace $\left(a_{1}, a_{2}, \ldots, a_{d}\right)$ by $\left(-a_{1}, a_{2}, \ldots, a_{d}\right)$. Now Lemma 2.4 says, $\left(b_{1}, \ldots, b_{d}\right)$ is obtained from $\left(a_{1}, \ldots, a_{d}\right)$ by a sequence of elementary operations. Each elementary operation relates the two sets of generators as in (a), with $N$ $=$ elementary matrix $\in \mathrm{SL}(\mathbb{Z})$. Multiplying these matrices we obtain the desired conclusion. 


\section{3. $H$-slices.}

In this section we establish several simple properties of slices on a $G$-variety $X$. Note that we do not assume that $X$ is affine or that $G$ is reductive. (Under these assumptions, one can prove quite a bit more than we do here; see $[\mathbf{L u}]$ or $[\mathbf{P V 1}$, Section 6].)

Definition 3.1. Let $G$ be an algebraic group and $X$ be a $G$-variety. We will call a locally closed subvariety $V$ of $X$ a slice at $x \in V$ if $X$ and $V$ are smooth at $x$ and $T_{x}(X)=T_{x}(G x) \oplus T_{x}(V)$. (Here $T_{x}(X)$ denotes the tangent space to $X$ at $x$.) If, moreover, $V$ is invariant under the action of a subgroup $H$ of $\operatorname{Stab}(x)$, we will refer to $V$ as an $H$-slice.

Remark 3.2. Note that since $V$ is smooth at $x$, we may replace $V$ by its (unique) irreducible component passing through $x$ and thus assume that $V$ is irreducible.

Example 3.3. Let $G$ be an algebraic group, $H$ be an algebraic subgroup of $G$ and $V$ be an $H$-variety. Recall that the homogeneous fiber product $X=G *_{H} X$ is defined as the geometric quotient $X=(G \times V) / H$, where $H$ acts on $G \times V$ by $h(g, v)=\left(g h^{-1}, h v\right)$. (This geometric quotient exists under mild assumptions on $V$; in particular, it exists whenever $V$ is quasiprojective; see [PV1, Theorem 4.19].) Note that $X=G *_{H} V$ is naturally a $G$-variety, where $G$ acts by left multiplication on the first factor; the details of this construction can be found in [PV1, Section 4.8].

The points of $X$ are in 1-1 correspondence with $H$-orbits in $G \times V$; we shall denote the point $x \in X$ corresponding to the $H$-orbit of $(g, v)$ in $G \times V$ by $x=[g, v]$. Let $\widetilde{V}$ be the image of the $H$-equivariant map $V \longrightarrow X$ given by $v \mapsto[1, v]$. With these notations, $\widetilde{V}$ is an $H$-slice for $X$ at $x=[1, v]$ for every smooth point $v$ of $V$; see [PV1, Proposition 4.22].

Lemma 3.4. Let $G$ be an algebraic group, $X$ be an irreducible $G$-variety, and $V$ be a slice at $x \in X$. Then $G V$ is dense in $X$.

Proof. Consider the map $\phi: G \times V \longrightarrow X$, given by $\phi(g, v)=g v$. The differential $d \phi_{(1, x)}: T_{1}(G) \times T_{x}(V) \longrightarrow T_{x}(X)$ is onto, since its image contains both $T_{x}(G x)$ and $T_{x}(V)$. Consequently, $d \phi$ is onto at a general point of $G \times V$. Thus $\phi$ is dominant, and the lemma follows.

Lemma 3.5. Let $G$ be an algebraic group, $H$ be a subgroup, $X$ be a $G$ variety, and $x$ is a smooth $H$-fixed point of $X$. If $H$ is reductive then $X$ has an $H$-slice at $x$.

Proof. Let $\mathcal{M}_{x, X}$ be the maximal ideal of the local ring of $X$ at $x$. Consider the natural $H$-equivariant linear maps $\mathcal{M}_{x, X} \longrightarrow T_{x}(X)^{*} \longrightarrow T_{x}(G x)^{*}$. Since $H$ is reductive these maps have $H$-equivariant splittings as maps of $k$-vector spaces. Thus we can choose a local coordinate system $u_{1}, \ldots, u_{n}$ 
in $\mathcal{M}_{x, X}$ such that $\operatorname{Span}_{k}\left(u_{1}, \ldots, u_{d}\right)$ is an $H$-invariant $k$-vector subspace of $\mathcal{M}_{x, X}$ and $u_{1}, \ldots, u_{d}$ (restricted to $G x$ ) form a local coordinate system in $\mathcal{O}_{x, G x} .($ Here $n=\operatorname{dim}(X)$ and $d=\operatorname{dim}(G x)$.)

Note that $u_{1}, \ldots, u_{n}$ are regular functions in some $H$-invariant open neighborhood of $x$. In this neighborhood a slice $V$ with desired properties is given by $u_{1}=\cdots=u_{d}=0$.

Recall that a $G$-variety $X$ is called generically free if $\operatorname{Stab}(x)=\{1\}$ for $x$ in general position in $X$.

Proposition 3.6. Let $G$ be an algebraic group, $H$ be a reductive subgroup, $X$ be a generically free $G$-variety and $x$ be a smooth $H$-fixed point of $X$. Then $H$ acts faithfully on $T_{x}(X) / T_{x}(G x)$.

Proof. Let $X_{0}$ be the unique component of $X$ passing through $x$ and $G_{0}$ be the subgroup of all elements of $G$ that preserve $X_{0}$. Note that $G_{0}$ has finite index in $G$ and $H \subset G_{0}$. After replacing $X$ by $X_{0}$ and $G$ by $G_{0}$, we may assume $X$ is irreducible.

We now argue by contradiction. Assume the kernel $K$ of the $H$-action on $T_{x}(X) / T_{x}(G x)$ is nontrivial. Since $K$ is a normal subgroup of $H$ and $H$ is reductive, $K$ is not unipotent. Hence, we can find a nonidentity element $g \in K$ of finite order.

By Lemma $3.5 X$ has an $H$-slice $V$ at $x$. Since $T_{x}(V) \simeq T_{x}(X) / T_{x}(G x)$ as $H$-representations, $g$ acts trivially on $T_{x}(V)$. This implies that $g$ acts trivially on $V$; see, e.g., [RY1, Lemma 4.2]. On the other hand, by Lemma 3.4 $G V$ is dense in $X$; consequently, for every $x \in X$ in general position $\operatorname{Stab}(x)$ contains a conjugate of $g$. Thus the $G$-action on $X$ is not generically free, contradicting our assumption.

\section{Definition and first properties of $i(X, x, H)$.}

Throughout this section we shall make the following assumptions:

$\begin{array}{ll}G & \text { algebraic group } \\ H & \text { finite abelian subgroup of } G \text { of rank } r \\ X & G \text {-variety of dimension } \operatorname{dim}(G)+r \\ x & \text { smooth } H \text {-fixed point of } X \text { whose stabilizer is finite. }\end{array}$

Definition 4.1. The $H$-representation on $T_{x}(X) / T_{x}(G x)$ decomposes as a direct sum of $r$ characters $\chi_{1}, \ldots, \chi_{r} \in H^{*}$. We define

$$
i(X, x, H)=\chi_{1} \wedge \cdots \wedge \chi_{r} \in \bigwedge^{r}\left(H^{*}\right) .
$$

Since the collection of characters $\chi_{1}, \ldots, \chi_{r}$ is well-defined, up to reordering, $i(X, x, H)$ is well-defined in $\bigwedge^{r}\left(H^{*}\right)$, up to multiplication by -1 . Thus, properly speaking, $i(X, x, H)$ should be viewed as an element of the factor set $\bigwedge^{r}\left(H^{*}\right) / \sim$, where $w_{1} \sim w_{2}$ iff $w_{1}= \pm w_{2}$. By abuse of notation we will 
sometimes write $i(X, x, H) \in \bigwedge^{r}\left(H^{*}\right)$; in such cases it should be understood that $i(X, x, H)$ is only defined up to sign.

Remark 4.2. It is clear from the definition that if $V$ is an $H$-slice for $X$ at $x$ then $i(X, x, H)=i(V, x, H)$. In particular, in the setting of Example 3.3, if $V$ is an $r$-dimensional $H$-variety, $X=G *_{H} V$, and $v$ is a smooth $H$-fixed point of $V$ then $i(X,[1, v], H)=i(\widetilde{V},[1, v], H)=i(V, v, H)$.

Remark 4.3. Let $g$ be an element of the normalizer $N_{G}(H)$ and let $\phi_{g}$ be the automorphism of $H$ sending $h$ to $g h g^{-1}$. Then it is easy to see that $i(X, g x, H)=\left(\wedge^{r} \phi_{g}^{*}\right)(i(X, x, H))$, where $\wedge^{r} \phi_{g}^{*}$ is the automorphism of $\bigwedge^{r}\left(H^{*}\right)$ induced by $\phi_{g}$.

Example 4.4. Let $G=H$ be a finite abelian group of rank $r, \chi_{1}, \ldots, \chi_{r}$ be a generating set for $H^{*}$, and $V=\mathbb{A}^{r}$ be a faithful $r$-dimensional linear representation of $H$, given by

$$
h:\left(v_{1}, \ldots, v_{r}\right) \longrightarrow\left(\chi_{1}(h) v_{1}, \ldots, \chi_{r}(h) v_{r}\right) .
$$

Then the origin $0_{V}$ is the only $H$-fixed point of $V$, and Definition $4.1 \mathrm{im}-$ mediately implies $i\left(V, 0_{V}, H\right)=\chi_{1} \wedge \cdots \wedge \chi_{r}$. The extended $H$-action on $\bar{V}=\mathbb{P}^{r}$, given by

$$
h\left(v_{0}: v_{1}: \cdots: v_{d}\right)=\left(v_{0}: \chi_{1}(h) v_{1}: \cdots: \chi_{d}(h) v_{d}\right),
$$

has exactly $r+1$ fixed points:

$$
x_{0}=(1: 0: \cdots: 0), \ldots, x_{r}=(0: \cdots: 0: 1) .
$$

Note that $x_{0}=0_{V}$. We claim that, up to sign,

$$
i\left(\bar{V}, x_{j}, H\right)=i\left(V, x_{0}, H\right)=\chi_{1} \wedge \cdots \wedge \chi_{r}
$$

for $j=1, \ldots, r$. To prove this claim, say for $j=1$, note that $v_{0} / v_{1}, v_{2} / v_{1}$, $\ldots, v_{r} / v_{1}$ form an affine coordinate system on $\bar{V}$ near $x_{1}$. The $H$-action is diagonal in these coordinates, and the representation of $H$ on $T_{x_{1}}(\bar{V})$ is the direct sum of the characters $\chi_{1}^{-1}, \chi_{2} \chi_{1}^{-1}, \ldots, \chi_{r} \chi_{1}^{-1}$. Consequently, $i\left(\bar{V}, x_{1}, H\right)= \pm \chi_{1}^{-1} \wedge \chi_{2} \chi_{1}^{-1} \wedge \cdots \wedge \chi_{r} \chi_{1}^{-1}= \pm \chi_{1} \wedge \cdots \wedge \chi_{r}$, as claimed.

Lemma 4.5. Suppose $X$ is a generically free $G$-variety. Then $i(X, x, H)$ generates $\bigwedge^{r}\left(H^{*}\right)$ as a group.

Note that the statement of the lemma makes sense, even though $i(X, x, H)$ is only defined up to sign: If $a$ generates $\bigwedge^{r}\left(H^{*}\right)$ then so does $-a$.

Proof. By Proposition 3.6 $H$ acts faithfully on $T_{x}(X) / T_{x}(G x)$. Hence, the characters $\chi_{1}, \ldots, \chi_{r}$ introduced in Definition 4.1 generate $H^{*}$ as an abelian group, and the lemma follows. 


\section{5. $i(X, x, H)$ and birational morphisms.}

The purpose of this section is to prove the following:

Theorem 5.1. Let $G$ be an algebraic group of dimension $d, H$ be a finite abelian subgroup of $G$ of rank $r, f: X \longrightarrow Y$ be birational morphism of irreducible generically free $G$-varieties of dimension $d+r, x$ is a smooth $H$-fixed point of $X, y=f(x)$ is a smooth point of $Y$, and $\operatorname{Stab}(y)$ is finite. Then $i(X, x, H)=i(Y, y, H)$.

Case I: $G=H$. As a first step towards proving Theorem 5.1, we will consider the case where $G=H$ is a finite abelian group. In this case Theorem 5.1 can be restated as follows.

Proposition 5.2. Let $H$ be a finite abelian group, and $f: X \longrightarrow Y$ be a birational morphism of irreducible generically free $H$-varieties of dimension $r=\operatorname{rank}(H)$. Assume that $x$ is a smooth $H$-fixed point of $X$ and $y=f(x)$ is a smooth point of $Y$. Then $i(X, x, H)=i(Y, y, H)$.

Before proceeding with the proof of Proposition 5.2, we introduce some background material on the power series ring $k\left[\left[u_{1}, \ldots, u_{r}\right]\right]$.

Given $w \in k\left[\left[u_{1}, \ldots, u_{r}\right]\right]$ we shall denote by $\operatorname{lm}(w)$ the lowest degree monomial in $u_{1}, \ldots, u_{r}$ which enters into $w\left(u_{1}, \ldots, u_{r}\right)$ with a nonzero coefficient. Here "lowest degree" refers to a fixed lexicographic monomial order $\succ$ given by, say, $u_{1} \succ \cdots \succ u_{r}$.

Suppose $v_{1}, \ldots, v_{m}$ lie in the maximal ideal of $k\left[\left[u_{1}, \ldots, u_{r}\right]\right]$, i.e., $\operatorname{lm}\left(v_{i}\right) \succ$ 1 for any $i=1, \ldots, m$. Then we can substitute $v_{1}, \ldots, v_{m}$ into any power series $p \in k\left[\left[z_{1}, \ldots, z_{m}\right]\right]$; in other words, $p\left(v_{1}, \ldots, v_{m}\right)$ is a well-defined element of $k\left[\left[u_{1}, \ldots, u_{r}\right]\right]$. If $p=Z$ is a monomial in $k\left[\left[z_{1}, \ldots, z_{m}\right]\right]$ then clearly

$$
\operatorname{lm}\left(Z\left(v_{1}, \ldots, v_{m}\right)\right)=Z\left(\operatorname{lm}\left(v_{1}\right), \ldots, \operatorname{lm}\left(v_{m}\right)\right) .
$$

We shall write $\left\langle u_{1}, \ldots, u_{r}\right\rangle$ for the group of all Laurent monomials in $u_{1}, \ldots$, $u_{r}$ (here we allow negative exponents).

Lemma 5.3. Suppose $v_{1}, \ldots, v_{m} \in k\left[\left[u_{1}, \ldots, u_{r}\right]\right]$. If $\operatorname{lm}\left(v_{1}\right), \ldots, \operatorname{lm}\left(v_{m}\right)$ generate a rank $m$ subgroup $\Lambda$ in $\left\langle u_{1}, \ldots, u_{r}\right\rangle \simeq \mathbb{Z}^{r}$ then $\operatorname{lm}\left(p\left(v_{1}, \ldots, v_{m}\right)\right) \in$ $\Lambda$ for any $p \in k\left[\left[z_{1}, \ldots, z_{m}\right]\right]$.

Note that the conditions of the lemma imply $m \leq r$; only the case $m=r$ will be used in the subsequent application.

Proof. Suppose $p\left(z_{1}, \ldots, z_{m}\right)=\sum c_{Z} Z$, where $Z$ ranges over all monomials in $z_{1}, \ldots, z_{m}$ with nonnegative exponents and each $c_{Z} \in K$. By our assumption $\operatorname{lm}\left(v_{1}\right), \ldots, \operatorname{lm}\left(v_{m}\right)$ are (multiplicatively) linearly independent, i.e.,

$$
Z\left(\operatorname{lm}\left(v_{1}\right), \ldots, \operatorname{lm}\left(v_{m}\right)\right) \neq Z^{\prime}\left(\operatorname{lm}\left(v_{1}\right), \ldots, \operatorname{lm}\left(v_{m}\right)\right)
$$


for any two distinct monomials $Z$ and $Z^{\prime}$. Suppose $Z_{0}\left(\operatorname{lm}\left(v_{1}\right), \ldots, \operatorname{lm}\left(v_{m}\right)\right)$ is the lexicographically smallest monomial $\left(\right.$ in $\left.u_{1}, \ldots, u_{m}\right)$ of the form $Z\left(\operatorname{lm}\left(v_{1}\right)\right.$, $\left.\ldots, \operatorname{lm}\left(v_{m}\right)\right)$, with $c_{Z} \neq 0$. Then (5.1) tells us that

$$
\operatorname{lm}\left(Z\left(v_{1}, \ldots, v_{m}\right)\right) \succ \operatorname{lm}\left(Z_{0}\left(v_{1}, \ldots, v_{m}\right)\right)=Z_{0}\left(\operatorname{lm}\left(v_{1}\right), \ldots, \operatorname{lm}\left(v_{m}\right)\right)
$$

for any $Z \neq Z_{0}$ with $c_{Z} \neq 0$. Thus

$$
\operatorname{lm}\left(p\left(v_{1}, \ldots, v_{m}\right)\right)=\operatorname{lm}\left(Z_{0}\left(v_{1}, \ldots, v_{m}\right)\right)=Z_{0}\left(\operatorname{lm}\left(v_{1}\right), \ldots, \operatorname{lm}\left(v_{m}\right)\right) \in \Lambda,
$$

as claimed.

Proof of Proposition 5.2. Diagonalizing the action of $H$ on the cotangent space $T_{x}^{*}(X)$, we obtain a basis $\bar{u}_{1}, \ldots, \bar{u}_{r} \in T_{x}^{*}(X)$ such that $h \bar{u}_{i}=\chi_{i}(h) \bar{u}_{i}$ for every $h \in H$; here $\chi_{1}, \ldots, \chi_{r} \in H^{*}$. Since the $k$-linear map $\mathcal{M}_{x, X} \longrightarrow$ $\mathcal{M}_{x, X} / \mathcal{M}_{x, X}^{2}=T_{x}^{*}(X)$ has an $H$-invariant $k$-linear splitting, we can find a local system of parameters $u_{1}, \ldots, u_{r} \in \mathcal{M}_{x, X}$ such that

$$
h u_{i}=\chi_{i}(h) u_{i}
$$

for every $h \in H$ and $i=1, \ldots, r$. Similarly, we can find a local coordinate system $v_{1}, \ldots, v_{r} \in \mathcal{M}_{y, Y}$ for $Y$ at $y$ and $\eta_{1}, \ldots, \eta_{r} \in H^{*}$ such that

$$
h v_{i}=\eta_{i}(h) v_{i}
$$

for every $h \in H$ and $i=1, \ldots, r$. Clearly $i(X, x, H)=\chi_{1} \wedge \cdots \wedge \chi_{r}$ and $i(Y, y, H)=\eta_{1} \wedge \cdots \wedge \eta_{r}$.

We shall identify the elements $v_{1}, \ldots, v_{r}$ with their images in $\mathcal{O}_{x, X}$ under $f^{*}: \mathcal{O}_{y, Y} \hookrightarrow \mathcal{O}_{x, X}$. The $H$-action on $\mathcal{O}_{x, X}$ naturally extends to $k\left[\left[u_{1}, \ldots, u_{r}\right]\right]$; in view of (5.2) the leading term map $w \mapsto \operatorname{lm}(w)$ is $H$-equivariant. Suppose $\operatorname{lm}\left(v_{i}\right)=u_{1}^{a_{i 1}} \ldots u_{r}^{a_{i r}}$ for some nonnegative integers $a_{i j}$. Then (5.2) and (5.3) imply $\eta_{i}=\prod_{j} \chi_{i}^{a_{i j}}$, and thus, up to sign,

$$
i(Y, y, H)=\eta_{1} \wedge \cdots \wedge \eta_{r}=\operatorname{det}\left(a_{i j}\right) \chi_{1} \wedge \cdots \wedge \chi_{r}= \pm \operatorname{det}\left(a_{i j}\right) i(X, x, H) .
$$

There are two conclusions we can draw from this formula. First of all, by Lemma 4.5 we know that both $i(X, x, H)$ and $i(Y, y, H)$ generate $\bigwedge^{r}\left(H^{*}\right)$; thus $\operatorname{det}\left(a_{i j}\right) \neq 0$. Secondly, in order to prove the proposition, it is sufficient to show that

$$
\operatorname{det}\left(a_{i j}\right)= \pm 1 \text { in } \mathbb{Z}
$$

We now proceed with the proof of (5.4). Let $\left\langle u_{1}, \ldots, u_{r}\right\rangle$ be the free abelian multiplicative group generated by $u_{1}, \ldots, u_{r}$. Since $\operatorname{det}\left(a_{i j}\right) \neq 0$, the leading monomials $\operatorname{lm}\left(v_{1}\right), \ldots, \operatorname{lm}\left(v_{r}\right)$ generate a (free abelian) subgroup $\Lambda$ of rank $r$ in $\left\langle u_{1}, \ldots, u_{r}\right\rangle \simeq \mathbb{Z}^{r}$; in other words, $\Lambda$ has finite index in $\left\langle u_{1}, \ldots, u_{r}\right\rangle$. On the other hand, (5.4) holds if and only if $\Lambda=\left\langle u_{1}, \ldots, u_{r}\right\rangle$. It is therefore sufficient to prove that $u_{i} \in \Lambda$ for every $i=1, \ldots, r$.

Since $\mathcal{O}_{x, X}$ and $\mathcal{O}_{y, Y}$ have the same field of fractions, each $u_{i}$ can be written as $p / q$, where $p, q \in \mathcal{O}_{y, Y}-\{0\}$. Represent $p$ and $q$ by power series 
in $v_{1}, \ldots, v_{r}$. By Lemma $5.3 \operatorname{lm}\left(p\left(v_{1}, \ldots, v_{r}\right)\right)$ and $\operatorname{lm}\left(q\left(v_{1}, \ldots, v_{r}\right)\right)$ lie in $\Lambda$; thus taking the leading monomials on both sides of the equation

$$
q\left(v_{1}, \ldots, v_{r}\right) u_{i}=p\left(v_{1}, \ldots, v_{r}\right),
$$

we conclude that $u_{i} \in \Lambda$, as desired.

Case II: $G$ - arbitrary. We are now ready to finish the proof of Theorem 5.1. The idea is to replace $X$ and $Y$ by suitable $H$-slices, then appeal to Proposition 5.2.

Diagonalizing the $H$-action on $T_{y}^{*}(G y)$, we obtain a basis $\bar{v}_{1}, \ldots, \bar{v}_{d}$ such that $h \bar{v}_{i}=\alpha_{i}(h) \bar{v}_{i}$ for some characters $\alpha_{1}, \ldots, \alpha_{d}$ of $H$. Since the natural $H$-equivariant $k$-vector space maps $\mathcal{M}_{y, Y} \longrightarrow T_{y}^{*}(Y) \longrightarrow T_{y}^{*}(G y)$ have $H$ equivariant splittings, we can lift $\bar{v}_{1}, \ldots, \bar{v}_{d}$ to $v_{1}, \ldots, v_{d} \in \mathcal{M}_{y, Y}$ such that such that $h v_{i}=\alpha_{i}(h) v_{i}$ for each $h \in H$. In other words, $v_{1}, \ldots, v_{d}$ form a local coordinate system for $G y$ at $y$.

Since both $\operatorname{Stab}(x)$ and $\operatorname{Stab}(y)$ are finite, $d f_{x}: T_{x}(G x) \longrightarrow T_{y}(G y)$ is an isomorphism. Thus $f^{*}\left(v_{1}\right), \ldots, f^{*}\left(v_{r}\right)$ form a local coordinate system for $G x$ at $x$. Define $W \subset Y$ as the irreducible component of $\left\{v_{1}=\cdots=v_{d}=0\right\}$ passing through $y$ and $V \subset X$ as the irreducible component of $\left\{f^{*}\left(v_{1}\right)=\right.$ $\left.\cdots=f^{*}\left(v_{d}\right)=0\right\}$ passing through $x$. Then $W$ is an $H$-slice for $Y$ at $y$ and $V$ is an $H$-slice for $X$ at $x$.

Clearly $f(V) \subset W$, i.e., $f_{\mid V}: V \longrightarrow W$ is a well-defined morphism. We claim that $f_{\mid V}$ is, in fact, a birational morphism. Theorem 5.1 follows from this claim because

$$
i(X, x, H) \stackrel{\text { Remark }}{=} 4.2 i(V, x, H) \stackrel{\text { Proposition } 5.2}{=} i(W, y, H) \stackrel{\text { Remark }}{=} 4.2 i(Y, y, H) .
$$

To show that $f_{\mid V}$ is dominant, assume, to the contrary, that $\operatorname{dim}(f(V))<$ $r$. Since $X$ is irreducible, $G V$ is dense in $X$ by Lemma 3.4 and thus $\operatorname{dim}(Y)=$ $\operatorname{dim}(f(X))=\operatorname{dim}(f(G V))=\operatorname{dim}(G f(V)) \leq d+\operatorname{dim}(f(V))<d+r$, a contradiction.

It remains to show that $f_{\mid V}$ is generically $1-1$ on closed points. Since $f$ is a birational morphism (i.e., has degree 1), there exists a dense $G$-invariant open subset $Y_{0}$ of $Y$ such that for every $y_{0} \in Y_{0}, f^{-1}\left(y_{0}\right)$ is a single point in $X$. Since $G W$ is dense in $Y$ (by Lemma 3.4), $Y_{0} \cap W$ is a dense open subset of $W$. Thus a general point of $W$ has exactly one preimage in $X$. On the other hand, a general point of $W$ has at least $\operatorname{deg}\left(f_{\mid V}\right) \geq 1$ preimages in $X$. This shows that $\operatorname{deg}\left(f_{\mid V}\right)=1$, i.e., $f_{\mid V}$ is birational, as claimed.

\section{Proof of Theorem 1.1.}

In this section we deduce Theorem 1.1 from Theorem 5.1. Our proof relies on canonical resolution of singularities. (We remark that canonical resolution of singularities is not used elsewhere in this paper.)

We begin with two simple preliminary results. 
Lemma 6.1. Let $H$ be an algebraic group, $f: Z \longrightarrow X$ be a birational morphism of complete irreducible $H$-varieties and $X_{1}$ be an irreducible $H$ invariant codimension 1 subvariety of $X$. If $X_{1}$ passes through a normal point of $X$ then there exists an $H$-invariant irreducible codimension 1 subvariety $Z_{1} \subset Z$ such that $f_{\mid Z_{1}}: Z_{1} \longrightarrow X_{1}$ is a birational morphism.

Proof. Since $X_{1}$ contains a normal point of $X$, the rational map $f^{-1}: X-\rightarrow$ $Z$ is defined at a general point of $X_{1}$. Now $Z_{1}=$ the closure of $f^{-1}\left(X_{1}\right)$ in $Z$, has the desired properties.

Proposition 6.2. Let $H$ be a diagonalizable group, $\alpha: Z \longrightarrow X$ be a birational morphism of complete irreducible $H$-varieties and $x$ be a smooth $H$-fixed point of $X$. Then there exists an $H$-fixed point $z \in Z$ such that $\alpha(z)=x$.

This result can be established by the argument used in the proof of $[\mathbf{R Y} \mathbf{1}$, Proposition A.4], due to J. Kollár and E. Szabó. (In fact, if $H$ is a $p$-group then Proposition 6.2 follows from [RY1, Proposition A.4].) We give a simple self-contained proof below.

Proof. We argue by induction on $\operatorname{dim}(X)$. The base case, $\operatorname{dim}(X)=0$, is obvious. For the induction step, assume $\operatorname{dim}(X) \geq 1$. We claim that there exists codimension $1 H$-invariant irreducible subvariety $X_{1}$ such that $x$ is a smooth point of $X_{1}$. Arguing as we did at the beginning of the proof of Proposition 5.2, we see that there exists a nonzero element $u \in \mathcal{M}_{x, X}$ such that for every $h \in H$, hu $=\alpha(h) u$, where $\alpha$ is a character of $H$. Then the (locally closed) subvariety $\{u=0\}$ of $X$ is $H$-invariant and smooth at $x$. Hence, its unique irreducible component passing through $x$ is also $H$ invariant, and we can define $X_{1}$ as the closure of this irreducible component. This proves the claim.

Now by Lemma 6.1 there exists a codimension 1 irreducible $H$-invariant subvariety $Z_{1}$ of $Z$ such that $\alpha_{\mid Z_{1}}: Z_{1} \longrightarrow X_{1}$ is a birational morphism of $H$-varieties. Applying the induction assumption to this morphism, we construct $z \in Z_{1} \subset Z$ with desired properties.

We are now ready to complete the proof of Theorem 1.1. The idea is to construct a complete smooth model $Z$ of $X$ (or $Y$ ) that dominates them both, i.e., fits into a diagram

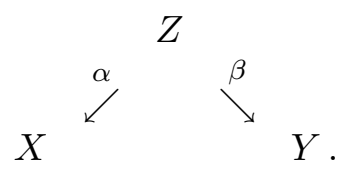

where $\alpha$ and $\beta$ are birational morphisms of $G$-varieties. If we find such a $Z$, Theorem 1.1 will easily follow. Indeed, by Proposition 6.2 there exists an 
$H$-fixed point $z \in Z$ such that $\alpha(z)=x$. Setting $y=\beta(z)$ and applying Theorem 5.1 to $\alpha$ and $\beta$, we conclude that $i(X, x, H)=i(Z, z, H)=i(Y, y, H)$, as desired.

It remains to construct $Z$. Let $W \subset X \times Y$ be the closure of the graph of a birational isomorphism $f$ between $X$ and $Y$. Then $W$ is a complete $G$-variety that dominates both $X$ and $Y$. In other words, $W$ satisfies all of our requirements for $Z$, with one exception: It may not be smooth. Let

$$
\pi: Z=W_{n} \stackrel{\pi_{n}}{\longrightarrow} \cdots \stackrel{\pi_{1}}{\longrightarrow} W_{0}=W,
$$

be the canonical resolution of singularities of $W$, as in [ $\mathbf{V}$, Theorem 7.6.1] or [BM, Theorem 1.6]. Here $Z$ is smooth, and each $\pi_{i}$ is a blowup with a smooth center; since these centers are canonically chosen, they are $G$ invariant. Thus the $G$ action can be lifted to $Z$ so that $\pi$ is a birational morphism of complete $G$-varieties. The smooth complete $G$-variety $Z$ constructed in this way has the desired properties.

Remark 6.3. An alternative construction of $Z$ is given by the equivariant version of Hironaka's theorem on elimination of points of indeterminacy (proved in $[\mathbf{R Y 4}]$ ), which asserts that for every rational map $f: X \rightarrow Y$ of $G$-varieties there exists a sequence of blowups

$$
\pi: Z=X_{n} \stackrel{\pi_{n}}{\longrightarrow} \cdots \stackrel{\pi_{1}}{\longrightarrow} X_{0}
$$

with smooth $G$-equivariant centers such that $f \pi: Z \longrightarrow Y$ is regular. The advantage of this approach is that it only uses Proposition 6.2 in the case where $\alpha$ is a single blowup with a smooth $G$-equivariant center (in which case the proof is immediate; see, e.g., [RY1, Lemma 5.1]). On the other hand, since the theorem on equivariant elimination of points of indeterminacy is itself deduced from canonical resolution of singularities in [RY4], we opted for a direct proof here.

Remark 6.4. A rational map $f: X \rightarrow Y$ (respectively, a morphism $f: X$ $\longrightarrow Y$ ) of reducible varieties is called a birational isomorphism (respectively, a birational morphism) if $X$ and $Y$ have irreducible decompositions $X_{1} \cup \cdots \cup$ $X_{n}$ and $Y_{1} \cup \cdots \cup Y_{n}$ such that $f$ restricts to a birational isomorphism $X_{i} \rightarrow$ $Y_{i}$ (respectively, a birational morphism) for each $i$. With this definition, the irreducibility assumption in Proposition 6.2 can be removed. Indeed, we can reduce to the case where $X$ and $Z$ are irreducible by replacing them with suitable irreducible components.

The irreducibility assumption in Theorem 1.1 (respectively, Theorem 5.1 and Proposition 5.2) can also be removed, if we assume $\operatorname{dim}_{x}(X)=d+r$ (respectively, $\operatorname{dim}_{x}(X)=d+r$ and $\operatorname{dim}_{x}(X)=r$ ). Indeed, if $X_{1}$ is the (necessarily unique) irreducible component of $X$ containing $x$ and $G_{1}=\{g \in$ $\left.G \mid g\left(X_{1}\right)=X_{1}\right\}$, then $H \subset G_{1},\left[G: G_{1}\right]<\infty$, and $i(X, x, H)=i\left(X_{1}, x, H\right)$, so that in each case we may replace $X, Y$, and $G$ by $X_{1}, Y_{1}$ and $G_{1}$. 
Remark 6.5. One may ask if the condition that $\operatorname{Stab}(x)$ is finite for every $x \in X^{H}$ (and similarly for $Y$ ) of Theorem 1.1 is ever satisfied. Indeed, if $H$ is contained in a torus $T$ of $G$ then the answer is "no", since $\operatorname{Stab}(x)$ is infinite for every $x \in X^{T} \subset X^{H}$, and $X^{T} \neq \emptyset$ by the Borel Fixed Point Theorem. On the other hand, if the centralizer $C_{G}(H)$ is finite, then we claim that every generically free $G$-variety $X$ has a birational model satisfying this condition. Indeed, by [RY1, Theorem 1.1] $X$ has a model with the property that the stabilizer of every point is of the form $U \rtimes D$, where $D$ is diagonalizable and $U$ is unipotent. Assume $x \in X^{H}$. By the Levi decomposition theorem, we may choose $D$ so that $D$ contains $H$. Now $[\mathbf{R Y 1}$, Lemma 7.3] tells us that $U=\{1\}$. Thus $\operatorname{Stab}(x)=D \subset C_{G}(H)$, which is finite, as claimed.

Examples of pairs $H \subset G$, where $G$ is a semisimple algebraic group and $H$ is an abelian subgroups of $G$ whose centralizer is finite can be found, e.g., in $[\mathbf{G r}],[\mathbf{S e 2}]$ or $[\mathbf{R Y 1}$, Section 8].

\section{A birational classification of linear representations.}

It is not difficult to see that Conjecture 1.2 fails if $G$ is a finite cyclic group of order $n=5$ or $\geq 7$. Indeed, let $V_{\omega}$ be the 1-dimensional representation of $G$ such that $\sigma$ acts on $V_{\omega}$ by the character $x \mapsto \omega x$, where $\omega$ is a primitive $n$th root of unity. Since any birational automorphism of $\mathbb{A}^{1}$ lifts to a regular automorphism of $\mathbb{P}^{1}$, it is easy to see that $V_{\omega}$ is birationally isomorphic to $V_{\omega^{\prime}}$ iff $\omega^{\prime}=\omega$ or $\omega^{\prime}=\omega^{-1}$. (The two $G$-fixed points in $\mathbb{P}^{1}$ are preserved in the former case and interchanged in the latter.) If $n=5$ or $\geq 7$, we can find two primitive $n$th roots of unity $\omega$ and $\omega^{\prime}$ such that $\omega^{\prime} \neq \omega^{ \pm 1}$, so that $V_{\omega}$ and $V_{\omega^{\prime}}$ are not birationally isomorphic. (P.I. Katsylo has informed us that this observation was independently made by E.A. Tevelev.)

In this section we will classify faithful linear representations of diagonalizable group $G$ up to birational equivalence and show that Conjecture 1.2 fails for a number of groups, both abelian and nonabelian. These results have the same general flavor as the observation in the previous paragraph but the arguments are more complicated due to the fact that we will be working with higher-dimensional varieties, rather than curves.

Representations of diagonalizable groups. Recall that every linear representation $V$ of $G$ decomposes as a sum of 1-dimensional character spaces ([Bo, Proposition III.8.2(d)]); if the associated characters of $G$ are $\chi_{1}, \ldots$, $\chi_{d}$, we shall write $V=\chi_{1} \oplus \cdots \oplus \chi_{d}$.

Theorem 7.1. Let $G$ be a diagonalizable group of rank $r$ and let $V=\chi_{1} \oplus$ $\cdots \oplus \chi_{d}$ and $W=\eta_{1} \oplus \cdots \oplus \eta_{d}$ be faithful d-dimensional linear representations of $G$. (In particular, $d \geq r$.) Then $V$ and $W$ are birationally isomorphic as $G$-varieties if and only if $\chi_{1} \wedge \ldots \wedge \chi_{d}= \pm \eta_{1} \wedge \cdots \wedge \eta_{d}$ in $\wedge^{d}\left(G^{*}\right)$. 
Proof. Since $G$ acts faithfully on $V$ and $W$, we have

$$
\left\langle\chi_{1}, \ldots, \chi_{d}\right\rangle=\left\langle\eta_{1}, \ldots, \eta_{d}\right\rangle=G^{*} \text {. }
$$

Assume $\chi_{1} \wedge \ldots \wedge \chi_{d}= \pm \eta_{1} \wedge \cdots \wedge \eta_{d}$. Then by Corollary 2.5 there exists an $N=\left(n_{i j}\right) \in \mathrm{GL}(\mathbb{Z})$ such that $\eta_{i}=\chi_{1}^{n_{1 i}} \ldots \chi_{d}^{n_{d i}}$. The desired birational isomorphism $V \rightarrow W$ is can now be explicitly given, in the natural coordinates on $V$ and $W$, by $\left(x_{1}, \ldots, x_{d}\right) \longrightarrow\left(y_{1}, \ldots, y_{d}\right)$, where $y_{i}=x_{1}^{n_{1 i}} \ldots x_{d}^{n_{d i}}$.

Conversely, suppose

$$
\chi_{1} \wedge \ldots \wedge \chi_{d} \neq \pm \eta_{1} \wedge \cdots \wedge \eta_{d} \text { in } \wedge^{d}\left(G^{*}\right) .
$$

We want to prove that $V$ and $W$ are not birationally isomorphic as $G$ varieties. Note that (7.2) is impossible if $d \geq r+1$, since in this case $\bigwedge^{d}\left(G^{*}\right)=(0)$; see Lemma 2.1(b). Thus we will assume from now on that $d=r=\operatorname{rank}(G)$. We will consider three cases.

Case 1. $G=\left(G_{m}\right)^{r}$ is a torus. In this case $\chi_{1} \wedge \ldots \wedge \chi_{r}$ and $\eta_{1} \wedge \cdots \wedge \eta_{r}$ are both generators of $\bigwedge^{r}\left(G^{*}\right)=\mathbb{Z}$, so that (7.2) is impossible.

Case 2. $G$ is a finite abelian group. The $G$-action on $V=\mathbb{A}^{r}$ (respectively $W=\mathbb{A}^{r}$ ) naturally extends to the projective space $\bar{V}=\mathbb{P}^{r}$ (respectively, $\bar{W}=\mathbb{P}^{r}$ ). Example 4.4 shows that for every $G$-fixed point $x \in \bar{V}, i(\bar{V}, x, G)= \pm \chi_{1} \wedge \cdots \wedge \chi_{r}$ and for every $G$-fixed point $y \in \bar{W}$, $i(\bar{W}, y, G)= \pm \eta_{1} \wedge \cdots \wedge \eta_{r}$. Thus in view of (7.2), Theorem 1.1 says that $\bar{V}$ and $\bar{W}$ (and, hence, $V$ and $W$ ) are not birationally isomorphic as $G$-varieties. Case 3. $G$ is a diagonalizable group but not a torus. Write $G=\mathbb{G}_{m}\left(n_{1}\right) \times$ $\cdots \times \mathbb{G}_{m}\left(n_{r}\right)$, as in (2.1). Since $G$ is not a torus, $n_{1} \geq 2$. Let $H=\mathbb{G}_{m}\left(n_{1}\right)^{r}=$ $\left(\mathbb{Z} / n_{1} \mathbb{Z}\right)^{r}$ be the $n_{1}$-torsion subgroup of $G$. It is sufficient to show that $V$ and $W$ are not birationally isomorphic as $H$-varieties; then they certainly cannot be birationally isomorphic as $G$-varieties. By Case 2, it is enough to show that

$$
\chi_{1}^{\prime} \wedge \ldots \wedge \chi_{r}^{\prime} \neq \pm \eta_{1}^{\prime} \wedge \cdots \wedge \eta_{r}^{\prime} \text { in } \wedge^{r}\left(H^{*}\right)
$$

where $\chi_{i}^{\prime}$ and $\eta_{j}^{\prime}$ are the characters of $H$ obtained by restricting $\chi_{i}$ and $\eta_{j}$ from $G$ to $H$. Note that the inclusion $\phi: H \hookrightarrow G$ induces a surjection $\phi^{*}: G^{*} \longrightarrow H^{*}$ of the dual group, which, in turn, induced a map of cyclic groups $\bigwedge^{r}\left(\phi^{*}\right): \bigwedge^{r}\left(G^{*}\right) \longrightarrow \bigwedge^{r}\left(H^{*}\right)$. Elementary group theory tells us that $G^{*}=\left(\mathbb{Z} / n_{1} \mathbb{Z}\right) \times \cdots \times\left(\mathbb{Z} / n_{r} \mathbb{Z}\right), H^{*}=\left(\mathbb{Z} / n_{1} \mathbb{Z}\right)^{r}, \phi^{*}: G^{*} \longrightarrow H^{*}$ is (componentwise) reduction modulo $n_{1}$, and $\Lambda^{r}\left(\phi^{*}\right)$ is the identity map $\bigwedge^{r}\left(G^{*}\right)=\mathbb{Z} / n_{1} \mathbb{Z} \stackrel{\simeq}{\longrightarrow} \mathbb{Z} / n_{1} \mathbb{Z}=\bigwedge^{r}\left(H^{*}\right)$. Applying $\bigwedge^{r}\left(\phi^{*}\right)$ to both sides of (7.2), we obtain (7.3), as desired.

Proof of Theorem 1.3. Let $G$ be a diagonalizable group $G$ of rank $r$ of the form (1.1), and let $V=\chi_{1} \oplus \cdots \oplus \chi_{d}$ and $W=\eta_{1} \oplus \cdots \oplus \eta_{d}$ be faithful $d$-dimensional linear representations of $G$. 
(a) If $d \geq r+1$ then $\wedge^{r}\left(G^{*}\right)=(0)$, so that $\chi_{1} \wedge \ldots \wedge \chi_{d}=0=\eta_{1} \wedge \cdots \wedge \eta_{d}$. Thus $V$ and $W$ are birationally isomorphic as $G$-varieties by Theorem 7.1.

From now on we will assume $d=r$. Note that in this case both $\chi_{1} \wedge \ldots \wedge \chi_{r}$ and $\eta_{1} \wedge \cdots \wedge \eta_{r}$ are generators of $\wedge^{r}\left(G^{*}\right)=\mathbb{Z} / n_{1} \mathbb{Z}$.

(b) Suppose $n_{1}=2$. Since $\bigwedge^{r}\left(G^{*}\right)=\mathbb{Z} / 2 \mathbb{Z}$ has only one generator, $\chi_{1} \wedge \ldots \wedge$ $\chi_{r}=\eta_{1} \wedge \cdots \wedge \eta_{r}$. Thus $V$ and $W$ are birationally isomorphic as $G$-varieties by Theorem 7.1 .

Now assume $n_{1}=0$. Then $\bigwedge^{r}\left(G^{*}\right)=\mathbb{Z}$. The only generators of this group are \pm 1 ; thus $\chi_{1} \wedge \ldots \wedge \chi_{r}= \pm \eta_{1} \wedge \cdots \wedge \eta_{r}$, and, once again, Theorem 7.1 tells us that $V$ and $W$ are birationally isomorphic.

(c) Suppose $n_{1} \geq 3$. By Theorem 7.1, birational isomorphism classes of $r$ dimensional linear representations of $H$ are in 1-1 correspondence with the generators of $\bigwedge^{r}\left(H^{*}\right) \simeq \mathbb{Z} / n_{1} \mathbb{Z}$ (as an additive group), modulo multiplication by -1 . Since $n_{r} \geq 3, a \neq-a$ for any generator $a$ of $\mathbb{Z} / n_{1} \mathbb{Z}$. Thus in this case the number of isomorphism classes of faithful $r$-dimensional $H$-representations is $\phi\left(n_{r}\right) / 2$, as claimed.

Further counterexamples to Conjecture 1.2. Theorem 1.3 shows that Conjecture 1.2 fails for many diagonalizable groups. We will now see that this conjecture also fails for some nonabelian finite groups.

Proposition 7.2. Let $n$ and $r$ be a positive integers, $P$ be a subgroup of $\mathrm{S}_{r}$, and $G=(\mathbb{Z} / n \mathbb{Z})^{r} \rtimes P$, where $P$ acts on $(\mathbb{Z} / n \mathbb{Z})^{r}$ by permuting the factors. Assume there exists an $m \in \mathbb{Z}$ such that $(m, n)=1$ and $m^{r} \not \equiv \pm 1(\bmod n)$. Then there exist two birationally inequivalent $r$-dimensional representations of $G$. In particular, Conjecture 1.2 fails for this group.

We remark that an integer $m$ satisfying the requirements of Proposition 7.2 always exists if the exponent of $U_{n}$ does not divide $2 r$; here $U_{n}$ is the (multiplicative) group of units in $\mathbb{Z} / n \mathbb{Z}$. In particular, $m$ exists if there is a prime power $p^{e}$ such that $p^{e} \mid n$ but $\phi\left(p^{e}\right)=(p-1) p^{e-1} \backslash 2 r$.

Proof. Let $\omega$ be a primitive $n$th root of unity. We define the $r$-dimensional representations $V$ and $W$ of $G$ as follows:

$$
\left(\left(a_{1}, \ldots, a_{r}\right), \sigma\right):\left(v_{1}, \ldots, v_{r}\right) \mapsto\left(\omega^{a_{1}} v_{\sigma^{-1}(1)}, \ldots, \omega^{a_{r}} v_{\sigma^{-1}(r)}\right)
$$

and

$$
\left(\left(a_{1}, \ldots, a_{r}\right), \sigma\right):\left(w_{1}, \ldots, w_{r}\right) \mapsto\left(\omega^{m a_{1}} w_{\sigma^{-1}(1)}, \ldots, \omega^{m a_{r}} w_{\sigma^{-1}(r)}\right) .
$$

Here $a_{1}, \ldots, a_{n} \in \mathbb{Z} / n \mathbb{Z}, \sigma \in P \subset \mathrm{S}_{r},\left(v_{1}, \ldots, v_{r}\right) \in V$ and $\left(w_{1}, \ldots, w_{r}\right) \in W$. It is easy to see that $V$ and $W$ are, indeed, well-defined faithful $r$-dimensional representations of $G$.

To prove the proposition it is sufficient to show that $V$ and $W$ are not birationally isomorphic as $(\mathbb{Z} / n \mathbb{Z})^{r}$-varieties. Let $\chi_{i}$ be the character of 
$(\mathbb{Z} / n \mathbb{Z})^{r}$ given by $\chi\left(a_{1}, \ldots, a_{r}\right)=\omega^{a_{i}}$. Then, as $\mathbb{Z} / n \mathbb{Z}$-representations, $V=$ $\chi_{1} \oplus \cdots \oplus \chi_{r}$ and $W=\chi_{1}^{m} \oplus \cdots \oplus \chi_{r}^{m}$. By our assumption

$$
\chi_{1}^{m} \wedge \cdots \wedge \chi_{r}^{m}=m^{r} \chi_{1} \wedge \cdots \wedge \chi_{r} \neq \pm \chi_{1} \wedge \cdots \wedge \chi_{r}
$$

in $\bigwedge^{r}\left(\mathbb{Z} / n \mathbb{Z}^{*}\right) \simeq \mathbb{Z} / n \mathbb{Z}$. Thus Theorem 7.1 tells us that $V$ and $W$ are not isomorphic as $(\mathbb{Z} / n \mathbb{Z})^{r}$-varieties (and hence, as $G$-varieties).

Remark 7.3. The same argument proves the following stronger result. Let $n_{1}, n_{2}, \ldots, n_{s}, r_{1}, \ldots, r_{s}$ be positive integers such that $n_{i}$ divides $n_{i+1}$ for $i=2, \ldots, r$, let $P_{i}$ be a subgroup of the symmetric group $\mathrm{S}_{r_{i}}$ and let $G_{i}=$ $\left(\mathbb{Z} / n_{i} \mathbb{Z}\right)^{r_{i}} \rtimes P_{i}$. Assume there exist integers $m_{1}, \ldots, m_{s}$ such that $\left(m_{i}, n_{i}\right)=$ 1 and $m_{1}^{r_{1}} \ldots m_{s}^{r_{s}} \not \equiv \pm 1\left(\bmod n_{1}\right)$. Then $G=\left(G_{m}\right)^{a} \times G_{1} \times \cdots \times G_{s}$ has two birationally inequivalent $\left(a+r_{1}+\cdots+r_{s}\right)$-dimensional representations. In particular, Conjecture 1.2 fails for any $G$ of this form.

Remark 7.4. The proof of Proposition 7.2 shows that $G=(\mathbb{Z} / n \mathbb{Z})^{r} \rtimes$ $P$ has at least $\left| \pm U_{n}^{r}\right| / 2$ birational isomorphism classes of $r$-dimensional representations. Here, as before, $U_{n}$ denotes the multiplicative group of units in the ring $\mathbb{Z} / n \mathbb{Z}$, and $\pm U_{n}^{r}$ denotes the subset of $U_{n}$ consisting of elements of the form $\pm m^{r}$, as $m$ ranges over $U_{n}$.

A similar estimate can be given for the number of birational isomorphism classes of $\left(a+r_{1}+\cdots+r_{s}\right)$-dimensional representations of the group $G$ in Remark 7.3. In particular, if $n_{1}=\cdots=n_{s}$ and $\left(r_{1}, \ldots, r_{s}\right)=1$ then there are at least $\phi\left(n_{1}\right) / 2$ such classes.

\section{Birational equivalence of quantum tori.}

In this section we will use the invariant $i(X, x, H)$ to classify $\mathrm{PGL}_{n}$-varieties (and consequently central simple algebras) of a certain form. In particular, we will prove Theorem 1.4.

Abelian subgroups of $\mathrm{PGL}_{n}$. Let $A$ be a finite abelian group of order $n$ and let $V=k[A]$ be the group ring of $A$. For $a \in A$ and $\chi \in A^{*}$ define $P_{a}, D_{\chi} \in \mathrm{GL}(V)$ by $P_{a}(b)=a b$ and $D_{\chi}(b)=\chi(b) b$ for every $b \in A$. It is easy to see that $D_{\chi} P_{a}=\chi(a) P_{a} D_{\chi}$. Thus if $p_{a}$ and $d_{\chi}$ denote the elements of PGL $(V)$ represented, respectively, by $P_{a}$ and $D_{\chi} \in \mathrm{GL}(V)$, then

$$
\begin{aligned}
& \phi: A \times A^{*} \hookrightarrow \operatorname{PGL}(V)=\mathrm{PGL}_{n} \\
&(a, \chi) \longrightarrow p_{a} d_{\chi}
\end{aligned}
$$

defines an embedding of $A \times A^{*}$ in $\mathrm{PGL}_{n}$.

Let $H$ be an abelian subgroup of $\mathrm{PGL}_{n}$. Then $H$ is naturally equipped with an alternating bilinear form $\omega_{H}: H \times H \longrightarrow \mu_{n}$ (cf. Definition 2.2(a)). Here $\mu_{n}$ is the group of $n$th roots of unity in $k$, identified with the center of $\mathrm{SL}_{n}$, and $\omega_{H}(a, b)=A B A^{-1} B^{-1}$, where $A$ and $B \in \mathrm{SL}_{n}$ represent $a$ and $b \in \mathrm{PGL}_{n}$ respectively. 
Lemma 8.1. Let $A$ be a finite abelian group of rank $r, H=\phi\left(A \times A^{*}\right)=$ $\left\{p_{a} d_{\chi} \mid a \in A, \chi \in A^{*}\right\}$ be the subgroup of $\mathrm{PGL}_{n}$ defined above. Then

(a) the elements of $P_{a} D_{\chi}$ span the matrix algebra $\mathrm{M}_{n}=\mathrm{M}_{n}(k)$ as a $k$ vector space; here as a ranges over $A$ and $\chi$ ranges over $A^{*}$, and

(b) the alternating bilinear form $\omega_{H}$ is symplectic (i.e., nondegenerate).

Let $g$ be an element of the normalizer $N_{\mathrm{PGL}_{n}}(H)$, and $\psi_{g}: H \longrightarrow H$ be conjugation by $g$. Then

(c) $\psi_{g}$ preserves $\omega_{H}$, and

(d) $\psi_{g}$ induces the identity $\operatorname{map} \bigwedge^{2 r}\left(H^{*}\right) \longrightarrow \bigwedge^{2 r}\left(H^{*}\right)$.

Proof.

(a) See [RY3, Lemma 3.2].

(b) See [RY2, Lemma 7.8].

(c) Choose $a$ and $b \in H \subset \mathrm{PGL}_{n}$ and lift them to $A$ and $B \in \mathrm{SL}_{n}$. Since $A B A^{-1} B^{-1}$ is a central element of $\mathrm{SL}_{n}$, we have

$$
\begin{aligned}
\omega_{H}\left(\psi_{g}(a), \psi_{g}(b)\right) & =\omega_{H}\left(g a g^{-1}, g b g^{-1}\right) \\
& =\left(g A g^{-1}\right)\left(g B g^{-1}\right)\left(g A^{-1} g^{-1}\right)\left(g B^{-1} g^{-1}\right) \\
& =g\left(A B A^{-1} B^{-1}\right) g^{-1}=A B A^{-1} B^{-1}=\omega_{H}(a, b),
\end{aligned}
$$

as claimed.

(d) Follows from (b), (c) and Lemma 2.3(b).

$\mathrm{PGL}_{n}$-varieties.

Proposition 8.2. Let $A$ be a finite abelian group of order $n$ and rank $r$ and let $H=\phi\left(A \times A^{*}\right)$ be the subgroup of $\mathrm{PGL}_{n}$ defined above. Suppose $V=\chi_{1} \oplus \cdots \oplus \chi_{2 r}$ and $W=\eta_{1} \oplus \cdots \oplus \eta_{2 r}$ are faithful representations of $H$. Then the following are equivalent:

(a) $\chi_{1} \wedge \cdots \wedge \chi_{2 r}= \pm \eta_{1} \wedge \cdots \wedge \eta_{2 r}$ in $\wedge^{2 r}\left(H^{*}\right)$,

(b) $V$ and $W$ are birationally isomorphic as $H$-varieties,

(c) $X=\mathrm{PGL}_{n} *_{H} V$ and $Y=\mathrm{PGL}_{n} *_{H} W$ are birationally isomorphic as $\mathrm{PGL}_{n}$-varieties.

Here $\mathrm{PGL}_{n} *_{H} V$ and $\mathrm{PGL}_{n} *_{H} W$ are homogeneous fiber products; see Example 3.3.

Proof. (a) and (b) are equivalent by Theorem 7.1. The implication (b) $\Longrightarrow$ (c) is obvious.

Thus we only need to show (c) $\Longrightarrow$ (a). The idea of the proof is to appeal to Theorem 1.1. We begin by observing that $X$ and $Y$ naturally embed as dense open subsets in projective varieties $\bar{X}=\left(\mathbb{P}\left(\mathrm{M}_{n}\right) \times \bar{V}\right) / H$ and $\bar{Y}=\left(\mathbb{P}\left(\mathrm{M}_{n}\right) \times \bar{W}\right) / H$ respectively. Here $\bar{V}=\mathbb{P}^{2 r}$ is the projective completion of $V=\mathbb{A}^{2 r} ; \mathrm{PGL}_{n}$ acts on $\mathbb{P}\left(\mathrm{M}_{n}\right) \times \bar{V}$ by left multiplication on 
the first factor; this action commutes with the $H$-action on $\mathbb{P}\left(\mathrm{M}_{n}\right) \times \bar{V}$ given by $h:(x, y) \longrightarrow\left(x h^{-1}, h y\right)$ and thus descends to the geometric quotient $\bar{X}=\left(\mathbb{P}\left(\mathrm{M}_{n}\right) \times \bar{V}\right) / H$. We shall denote the point $x \in \bar{X}$ corresponding to the orbit of $(g, v) \in \mathbb{P}\left(\mathrm{M}_{n}\right) \times \bar{V}$ by $[g, v]$. The $H$-variety $\bar{W}$ and the $\mathrm{PGL}_{n}$-variety $\bar{Y}$ are defined in a similar manner.

Our goal is to show that

(i) every $H$-fixed points of $\bar{X}$ is of the form $x=[g, v]$, where $g \in N_{\mathrm{PGL}_{n}}(H)$ and $v$ is an $H$-fixed point of $\bar{V}$, and for any such point $x$,

(ii) $\operatorname{Stab}(x)=H$ and

(iii) up to sign, $i(X, x, H)=\chi_{1} \wedge \cdots \wedge \chi_{2 r}$.

These assertions, in combination with Theorem 1.1, will prove that if $X$ and $Y$ (and hence, $\bar{X}$ and $\bar{Y}$ ) are birationally isomorphic then $\chi_{1} \wedge \cdots \wedge \chi_{2 r}=$ $\pm \eta_{1} \wedge \cdots \wedge \eta_{2 r}$, i.e., (c) $\Longrightarrow$ (a).

To prove (i), assume $x=[g, v]$ is an $H$-fixed point of $\bar{X}$ for some $g \in$ $\mathbb{P}\left(\mathrm{M}_{n}\right)$ and $v \in \bar{V}$. This means that for every $h \in H$ there exists an $h^{\prime} \in H$ such that $(h g, v)=\left(g h^{\prime},\left(h^{\prime}\right)^{-1} v\right)$ in $\mathbb{P}\left(\mathrm{M}_{n}\right) \times \bar{V}$. Equivalently, $h g=g h^{\prime}$ and $\left(h^{\prime}\right)^{-1} v=v$.

Consider the vector space $k^{n}$ of $(n \times 1)$-row vectors. The multiplication by $g$ on the right yields a linear map $k^{n} \longrightarrow k^{n}$; let $\operatorname{RKer}(g)$ be the kernel of this map. Note that since $g \in \mathbb{P}\left(\mathrm{M}_{n}\right)$, this linear map is only defined up to a nonzero constant multiple but $\operatorname{RKer}(g)$ is well-defined.

The equality $h g=g h^{\prime}$ implies that $\operatorname{RKer}(g)$ is an $H$-invariant subspace of $k^{n}$ with respect to the right action of $H$; again, as $H \subset \mathrm{PGL}_{n}$, the right multipication by an element $h \in H$ is a linear map $k^{n} \longrightarrow k^{n}$ defined up to a nonzero constant multiple, but the notion of $H$-invariance of a linear subspace of $k^{n}$ is well-defined.

Now recall that by Lemma 8.1(a) the $n^{2}$ elements of the form $P_{a} D_{\chi}$ which represent the elements of $H \subset \mathrm{PGL}(V)=\mathrm{PGL}_{n}$ in $\mathrm{GL}(V)=\mathrm{GL}_{n}$ span $\mathrm{M}_{n}$ as a $k$-vector space. Thus the only $H$-invariant subspaces of $k^{n}$ are the ones that are invariant under all of $\mathrm{M}_{n}$, namely $k^{n}$ and (0). If $\operatorname{RKer}(g)=k^{n}$ then $g$ is the zero matrix, which is impossible since $g \in \mathbb{P}\left(\mathrm{M}_{n}\right)$. Thus we conclude that $\operatorname{RKer}(g)=(0)$. This means that $g$ is nonsingular, i.e., $g \in \mathrm{PGL}_{n}$. Now we can rewrite $h g=g h^{\prime}$ as $g^{-1} h g=h^{\prime} \in H$; this shows that $g \in N_{\mathrm{PGL}_{n}}(H)$. Moreover, as $h$ ranges over $H, h^{\prime}=g^{-1} h g$ also ranges over all of $H$. Thus the equality $\left(h^{\prime}\right)^{-1} v=v$ implies that $v$ is an $H$-fixed point of $\bar{V}$. This proves (i).

From now on let $x=[g, v]$ be an $H$-fixed point of $\bar{X}$, where $g \in N_{\mathrm{PGL}_{n}}(H)$ and $v$ is an $H$-fixed point of $\bar{V}$.

To prove (ii), assume $g^{\prime} \in \operatorname{Stab}(x)$, i.e., $g^{\prime}[g, v]=[g, v]$. Then $g^{\prime} g=g h^{\prime}$ for some $h^{\prime} \in H^{\prime}$. Since $g \in N_{\mathrm{PGL}_{n}}(H)$, we conclude that $g^{\prime}=g h g^{-1} \in H$, as desired. 
To prove (iii), first note that $i(\bar{X},[g, v], H)=\wedge^{2 r} \psi_{g}^{*}(i(\bar{X},[1, v], H))$, where $\psi_{g}: H \longrightarrow H$ is conjugation by $g \in N_{\mathrm{PGL}_{n}}(H)$ and $\wedge^{2 r}\left(\psi_{g}^{*}\right)$ is the automorphism of $\bigwedge^{2 r}\left(H^{*}\right)$ induced by $\psi_{g}$; see Remark 4.3. By Lemma 8.1(d) $\wedge^{2 r} \psi_{g}^{*}$ is the identity automorphism. Thus $i(\bar{X},[g, v], H)=i(\bar{X},[1, v], H)$. On the other hand, by Remark $4.2 i(\bar{X},[1, v], H)=i(\bar{V}, v, H)$. Finally, recall that for any $v \in \bar{V}^{H}, i(\bar{V}, v, H)=i\left(V, 0_{V}, H\right)=\chi_{1} \wedge \cdots \wedge \chi_{2 r}$; see Example 4.4. In summary,

$$
i(\bar{X},[g, v], H)=i(\bar{X},[1, v], H)=i(\bar{V}, v, H)=\chi_{1} \wedge \cdots \wedge \chi_{2 r},
$$

as claimed.

Remark 8.3. The exceptional group $E_{8}$ has a nontoral subgroup $H$ isomorphic to $(\mathbb{Z} / 5 \mathbb{Z})^{3}$; see $[\mathbf{G r}$, Lemma 10.3]. Modifying the proof of Proposition 8.2, we can show the following:

Let $V=\chi_{1} \oplus \chi_{2} \oplus \chi_{3}$ and $W=\eta_{1} \oplus \eta_{2} \oplus \eta_{3}$ be faithful 3-dimensional representations of $H$, where $\chi_{i}$ and $\eta_{j}$ are characters of $H$. Then the following are equivalent:

(a) $\chi_{1} \wedge \chi_{2} \wedge \chi_{3}= \pm \eta_{1} \wedge \eta_{2} \wedge \eta_{3}$ in $\wedge^{3}\left(H^{*}\right) \simeq \mathbb{Z} / 5 \mathbb{Z}$,

(b) $V$ and $W$ are birationally isomorphic as $H$-varieties, and

(c) $E_{8} *_{H} V$ and $E_{8} *_{H} W$ are birationally isomorphic as $E_{8}$-varieties.

In particular, there are exactly two birational isomorphism classes of $E_{8}$ varieties of the form $E_{8} *_{H} V$, where $V$ is a faithful 3-dimensional representation of $H$ : One corresponds to \pm 1 , and the other to \pm 2 in $\mathbb{Z} / 5 \mathbb{Z} \simeq \bigwedge^{3}\left(H^{*}\right)$.

Remark 8.4. Note that the $\mathrm{PGL}_{n}$-varieties $X=\mathrm{PGL}_{n} *_{H} V$ and $Y=$ $\mathrm{PGL}_{n} *_{H} W$ of Proposition 8.2, are stably isomorphic (as $\mathrm{PGL}_{n}$-varieties). In fact, $X \times \mathbb{A}^{1} \simeq Y \times \mathbb{A}^{1}$ because $X \times \mathbb{A}^{1}=\mathrm{PGL}_{n} * H\left(V \times \mathbb{A}^{1}\right), Y \times \mathbb{A}^{1}=$ $\mathrm{PGL}_{n} *_{H}\left(W \times \mathbb{A}^{1}\right)$, and $V \times \mathbb{A}^{1} \simeq W \times \mathbb{A}^{1}$ as $H$-varieties by Theorem 7.1. For the same reason $X \times \mathbb{A}^{1}$ and $Y \times \mathbb{A}^{1}$ are isomorphic $E_{8}$-varieties, if $X$ and $Y$ are as in Remark 8.3.

Proof of Theorem 1.4. Recall that birational isomorphism classes of generically free irreducible $\mathrm{PGL}_{n}$-varieties $X$ with $k(X)^{\mathrm{PGL}_{n}}=K$ are in 1-1 correspondence with central simple algebras of degree $n$ over $K$; see e.g., [Se1, X.5] or [RY2, Section 3].

In particular, by [RY3, Lemma 4.2], the algebra $Q\left(\omega_{1}, \ldots, \omega_{r}\right)$ of Theorem 1.4 corresponds to the variety $X=\mathrm{PGL}_{n} *_{H} V$, where $V$ is a faithful $2 r$-dimensional representations of $H=A \times A^{*}$ constructed as follows. Choose a set of generators $a_{1}, \ldots, a_{r}$ for $A=\mathbb{Z} / n_{1} \mathbb{Z} \times \cdots \times \mathbb{Z} / n_{r} \mathbb{Z}$ and a "dual" set of generators $\chi_{1}, \ldots, \chi_{r}$ for $A^{*}$ so that

$$
\chi_{i}\left(a_{j}\right)= \begin{cases}1 & \text { if } i \neq j \\ \omega_{i} & \text { if } i=j\end{cases}
$$


Now note that each $a \in A$ defines a character of $H=A \times A^{*}$ by $(b, \eta) \mapsto \eta(a)$. Similarly, each $\chi \in A^{*}$ gives rise to a character $H=A \times A^{*} \longrightarrow k^{*}$ via $(b, \eta) \mapsto \chi(b)$; we shall denote these characters by $c(a)$ and $c(\chi)$ respectively. In these notations,

$$
V=c\left(a_{1}\right) \oplus \cdots \oplus c\left(a_{r}\right) \oplus c\left(\chi_{1}\right)^{-1} \oplus \cdots \oplus c\left(\chi_{r}\right)^{-1},
$$

see [RY3, Proof of Lemma 4.2].

Similarly the $\mathrm{PGL}_{n}$-variety associated to $Q\left(\omega_{1}^{m_{1}}, \ldots, \omega_{r}^{m_{r}}\right)$ is $Y=\mathrm{PGL}_{n} * H$ $W$, where $W=c\left(a_{1}^{\prime}\right) \oplus \cdots \oplus c\left(a_{r}^{\prime}\right) \oplus c\left(\chi_{1}^{\prime}\right)^{-1} \oplus \cdots \oplus c\left(\chi_{r}^{\prime}\right)^{-1}$. Here $a_{1}^{\prime}, \ldots, a_{r}^{\prime}$ are generators of $A$ and $\chi_{1}^{\prime}, \ldots, \chi_{r}^{\prime}$ are generators of $A^{*}$ such that

$$
\chi_{i}^{\prime}\left(a_{j}^{\prime}\right)= \begin{cases}1 & \text { if } i \neq j \\ \omega_{i}^{m_{i}} & \text { if } i=j .\end{cases}
$$

A natural choice for $a_{i}^{\prime}$ and $\chi_{i}^{\prime}$ is $a_{i}^{\prime}=a_{i}$ and $\chi_{i}^{\prime}=\chi_{i}^{m_{i}}$, so that

$$
W=c\left(a_{1}\right) \oplus \cdots \oplus c\left(a_{r}\right) \oplus c\left(\chi_{1}\right)^{-m_{1}} \oplus \cdots \oplus c\left(\chi_{r}\right)^{-m_{r}} .
$$

As we mentioned above, $Q\left(\omega_{1}, \ldots, \omega_{r}\right)$ and $Q\left(\omega_{1}^{m_{1}}, \ldots, \omega_{r}^{m_{r}}\right)$ are isomorphic as $k$-algebras iff their associated $\mathrm{PGL}_{n}$-varieties, $X=\mathrm{PGL}_{n} * H$ and $Y=\mathrm{PGL}_{n} *_{H} W$, are birationally isomorphic. By Proposition $8.2 X$ and $Y$ are birationally isomorphic iff

$$
\begin{aligned}
& c\left(a_{1}\right) \wedge \cdots \wedge c\left(a_{r}\right) \wedge c\left(\chi_{1}\right)^{-1} \wedge \cdots \wedge c\left(\chi_{r}\right)^{-1} \\
& = \pm c\left(a_{1}\right) \wedge \cdots \wedge c\left(a_{r}\right) \wedge c\left(\chi_{1}\right)^{-m_{1}} \wedge \cdots \wedge c\left(\chi_{r}\right)^{-m_{r}} \text { in } \wedge^{2 r}\left(H^{*}\right) \simeq \mathbb{Z} / n_{r} \mathbb{Z} .
\end{aligned}
$$

The last condition is equivalent to $m_{1} \ldots m_{r}= \pm 1\left(\bmod n_{1}\right)$.

\section{References}

[BM] E. Bierstone and P.D. Milman, Canonical desingularization in characteristic zero by blowing up the maximum strata of a local invariant, Invent. Math., 128(2) (1997), 207-302, MR 98e:14010, Zbl 0896.14006.

[Bo] A. Borel, Linear Algebraic Groups, second edition, Springer-Verlag, 1991, MR 92d:20001, Zbl 0726.20030.

[DF] D.S. Dummit and R.M. Foote, Abstract Algebra, second edition, Prentice Hall, 1999, MR 92k:00007, Zbl 0943.00001.

[Gr] R.L. Griess, Jr., Elementary abelian p-subgroups of algebraic groups, Geom. Dedicata, 39 (1991), 253-305, MR 92i:20047, Zbl 0733.20023.

[K] P. Katsylo, On the birational classification of linear representations, Max Planck Institute für Mathematik, preprint MPI-92-1 (1992).

[Lang] S. Lang, Algebra, Addison-Wesley, 1965, MR 33 \#5416, Zbl 0193.34701.

[Lo1] M. Lorenz, Group rings and division rings, in 'Methods in Ring Theory', edited by F. van Oystaeyen, (1984), 265-280, MR 86d:16016, Zbl 0542.16009.

[Lo2] _ _ Division algebras generated by finitely generated nilpotent groups, J. Algebra, 85 (1983), 368-381, MR 85i:16014, Zbl 0527.16008. 
[Lu] D. Luna, Slices étales, Bull. Soc. Math. France, Mémoire, 33 (1973), 81-105, MR 49 \#7269, Zbl 0286.14014.

[P] V.L. Popov, Sections in Invariant Theory, Proceedings of the Sophus Lie Memorial Conference, Scandinavian University Press, (1994), 315-362, MR 98d:14058.

[PV1] V.L. Popov and E.B. Vinberg, Invariant Theory, in 'Encyclopedia of Math. Sciences', 55, Algebraic Geometry IV, edited by A.N. Parshin and I.R. Shafarevich, Springer-Verlag, 1994, MR 92d:14010

[PV2] _ Some open problems in invariant theory, Contemporary Math., 131(2) (1992), 485-497, MR 93f:14026, Zbl 0783.14028.

[RY1] Z. Reichstein and B. Youssin, Essential dimensions of algebraic groups and a resolution theorem for G-varieties, with an appendix by J. Kollár and E. Szabó, Canadian J. Math., 52(5) (2000), 1018-1056, MR 2001k:14088.

[RY2] _ Splitting fields of G-varieties, Pacific J. Math., 200(1) (2001), 207-249, CMP 1863413.

[RY3] _ Conditions satisfied by characteristic polynomials in fields and division algebras, J. Pure Appl. Algebra, 166 (2002), 165-189, CMP 1868544.

[RY4] Equivariant resolution of points of indeterminacy, to appear in Proc. Am. Math. Soc.

[Se1] J.-P. Serre, Local Fields, Springer-Verlag, 1979, MR 82e:12016, Zbl 0423.12016.

[Se2] - Sous-groupes finis des groupes de Lie, Séminaire Bourbaki, 1998-99, 864, Juin 1999, MR 2001i:20075.

[TA] J.P. Tignol and S.A. Amitsur, Symplectic modules, Israel J. Math., 54(3) (1986), 266-290, MR 87m:16042, Zbl 0599.20086.

[V] O.E. Villamayor U., Patching local uniformizations, Ann. Scient. Éc. Norm. Sup., 4e série, 25 (1992), 629-677, MR 93m:14012, Zbl 0782.14009.

Received July 13, 2000 and revised February 16, 2001. Z. Reichstein was partially supported by NSF grant DMS-9801675

Department of Mathematics

Oregon State University

Corvallis, OR 97331

Department of Mathematics

UNIVERSiTy OF BRITISH COLUMBIA

Vancouver, B.C., Canada V6T $1 Z 2$

E-mail address: reichst@math.ubc.ca

Department of Mathematics and Computer Science

UNIVERSITY OF THE NEGEV

BE'ER SHEVA', ISRAEL

HASHOFAR 26/3

MA'Ale Adumim, IsRael

E-mail address: youssin@math.huji.ac.il 